\title{
Wie befreien wir uns aus der digitalen Zwangsjacke?
}

\section{Yvonne Gilli}

Dr. med., Mitglied des FMH-Zentralvorstandes, Departementsverantwortliche Digitalisierung/eHealth

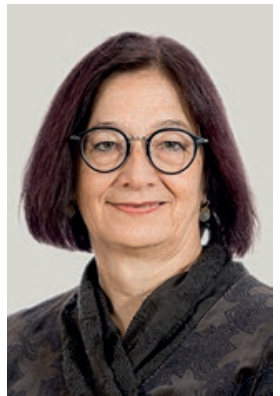

Die Chancen der Digitalisierung sollten wir endlich anpacken und digitale Werkzeuge zeitgemäss nutzen, sagen uns Politikerinnen und Behörden, und drohen mit Zwang. Die kalte Dusche folgte bereits diesen Sommer im Parlament mit der Koppelung der Berufsausübungsbewilligung an die Verpflichtung der Praxisärztinnen, am elektronischen Patientendossier gemäss Gesetz teilzunehmen. Weitere Verpflichtungen erwarten uns. Auch über die Medien verbreitete undifferenzierte oder sogar falsche Aussagen können den Druck seitens Politik auf die Ärzteschaft erhöhen: «Ärzte melden CoronaFälle per Fax» ${ }^{1}$, oder «Schweizer Ärzte melden nicht einmal jeden zweiten Corona-Fall $»^{2}$. Ersteres wurde uns von Behördenseite vorgegeben, Letzteres entspricht nicht der Wahrheit. Jedoch sind Überregulierung und Schuldzuweisungen bekannte strategische Instrumente, um mittels Kontrolle und Ablenkung die Verantwortung zu delegieren.

Ein Blick zurück auf die «lessons learned» aus anderen Ländern lohnt sich deshalb. Unter dem Titel «the HITECH Era in Retrospect» ${ }^{3}$ veröffentlichte das New England Journal of Medicine eine exzellente Zusammenfassung der Entwicklung in den USA. Mindestens zwei Erkenntnisse sind für die Schweiz wichtig: Die Digitalisierung im Gesundheitswesen ist ein gigantischer Treiber wirtschaftlichen Wachstums; und für die nutzenbringende Anwendung braucht es Anreize, welche «Herz und Geist» der Ärztinnen und Ärzte ansprechen. Letzteres kann nur erreicht werden, wenn die Ärzteschaft den digitalen Praxisalltag mitgestalten kann.

Im Winter 2019 besuchte eine Gruppe von Ärztinnen die digitale Vorreiternation in Europa: Estland. Neben eindrücklichen Beispielen, wie digitale Hilfsmittel, welche die Qualität ärztlichen Handelns in Notfallund Intensivmedizin unterstützen, zeigte ein Einblick in die dortige Praxistätigkeit, dass für die Triage in der ärztlichen Grundversorgung weder ein Chatbot noch ein digital unterstütztes telemedizinisches Angebot genutzt wurde. Es war schlicht das persönliche Telefongespräch zwischen der Praxisassistentin und dem Patienten, welches die effizienteste Triage darstellte. Die Aufgabe der Politik kann es deshalb nicht sein, den professionellen Handlungsspielraum von Ärztinnen und Ärzten mittels einer Regulierungskaskade einzuengen. Sie soll die Rahmenbedingungen schaffen, die es der Ärzteschaft ermöglichen, digitale Technologien im Berufsalltag zu nutzen. Dazu gehören staatliche Investitionen in die digitale Infrastruktur und einheitliche Standards zu Datenschutz, Datensicherheit und Interoperabilität. In der Folge können die jeweiligen Organisationen der Gesundheitsberufe ihre Mitglieder in der Umsetzung unterstützen.

Die politischen Verantwortlichen in der Schweiz haben das Pferd am Schwanz aufgezäumt, indem den Gesundheitsfachpersonen als Erstes ein bis ins letzte Detail behördlich reguliertes Produkt, das elektronische Patientendossier (EPD), "top down" verordnet wird. So verstanden ist es nicht erstaunlich, dass gerade dieses Produkt 2019 mit seiner zentralen Architektur bei fehlender Ende-zu-Ende-Verschlüsselung den «Big Brother Award» $»^{4}$ gewann. Unter diesen Voraussetzungen ergeben sich zwei Aufgaben für die Ärzteschaft: sich erstens einen möglichst grossen beruflichen Freiraum zu schaffen unter dieser «digitalen Zwangsjacke», und zweitens in der Digitalisierung ihres Berufsalltages die Führungsrolle zu übernehmen.

Ersteres gelingt u.a. durch die interprofessionelle Arbeitsgruppe IPAG, welche Standards setzt zu den Inhalten im EPD, oder durch die Gründung und Nutzung ärzteeigener Gefässe wie die AD Swiss EPD-Gemeinschaft.

Letzteres braucht Kompetenz und eine stark intrinsisch geprägte Berufsidentität. Beides ist mit Beginn der beruflichen Karriere vorhanden. Mit den Worten des Direktors des Bachelorstudienganges in Medizin an der ETH formuliert: «Wir bilden Mediziner aus mit einem besonderen Profil - Ärztinnen und Ärzte mit Spezialwissen in Naturwissenschaften und Technologie, die dank ihrem Rüstzeug auch Entwicklungen im Bereich der digitalen Medizin in die klinische Praxis umsetzen können». ${ }^{5}$ Damit diese kreative Kraft im heute überregulierten Berufsalltag nicht verloren geht, braucht es nicht nur jede einzelne Ärztin, sondern das Kollektiv der Ärzteschaft, welche über die einzelnen Fachrichtungen hinweg gemeinsam Grenzen setzen und Innovation gestalten kann. 